Arq. Bras. Med. Vet. Zootec., v.68, n.4, p.938-944, 2016

\title{
Tumor estromal gastrointestinal em cães: estudo clínico-anatomopatológico
}

\author{
[Gastrointestinal stromal tumors in dogs: a clinicopathologic study]

$$
\text { R.M. Leandro }{ }^{1} \text {, L.R.M. Sá }{ }^{2}
$$ \\ ${ }^{1}$ Universidade de São Paulo e Universidade Anhembi Morumbi - São Paulo, SP \\ ${ }^{2}$ Faculdade de Medicina Veterinária e Zootecnia - Universidade de São Paulo - São Paulo, SP
}

\section{RESUMO}

Os tumores estromais gastrointestinais (GIST) são incomuns e apresentam elevada similaridade histológica com as neoplasias de músculo liso e de nervo periférico. Este trabalho relata e caracteriza GIST em cães do ponto de vista epidemiológico, clínico-laboratorial, anatomopatológico e imuno-histoquímico, com base na análise retrospectiva de três cães, machos, com média de idade de 10 anos, variando de porte médio a grande e peso médio de $18.5 \pm 6 \mathrm{~kg}$. As alterações clínicas foram progressivas e inespecíficas de quadro gastrointestinal crônico, tais como prostração, anorexia, perda de peso e melena. Anemia não regenerativa e leucocitose foram os principais achados laboratoriais. Macroscopicamente, os tumores apresentavam média de $19 \mathrm{~cm}$ no eixo maior, localizados em jejuno e ceco, com a superfície ulcerada, e exibiam áreas de necrose. Na avaliação microscópica, os casos apresentaram proliferação de células fusiformes, arranjadas em feixes longos densamente celulares, que foram vimentina e CD117 positivas, com diferenciação neural (S100 positivo), muscular (actina e desmina positivas) e anaplásica, cuja morfologia caracteriza neoplasias malignas com sobrevida de um dia a 12 meses. O GIST deve ser um dos diagnósticos diferenciais a serem considerados entre as neoplasias mesenquimais do trato gastrointestinal que acometem cães, e o diagnóstico final necessita de imuno-histoquímica.

Palavras-chave: cão, C-KIT, intestino, GIST, imuno-histoquímica

\begin{abstract}
Gastrointestinal stromal tumors (GIST) are uncommon and show high histological similarity with smooth muscle and peripheral neuron tumors. This paper reported three cases of gastrointestinal stromal tumor (GIST) showing its clinical and laboratorial presentation, and gross, microscopical and immunohistochemical features. Clinical signs and symptoms of the animals studied were progressive and characterized by nonspecific chronic gastrointestinal disorders such as depression, anorexia, weight loss and melena. Non regenerative anemia and leukocytosis were the main laboratory findings. Grossly, tumors were $19 \mathrm{~cm}$ big, ulcerated and with necrotic areas. Microscopically, the tumors were malignant spindle cell proliferation with positivity to vimentine and CD117, with neural (S100+), myogenic (desmine + and smooth muscle actine+) and anaplasic differentiation. GIST should be one of the differential diagnoses to be considered among the mesenchymal neoplasms of the gastrointestinal tract of dogs.
\end{abstract}

Keywords: dog, C-KIT, intestine, GIST, immunohistochemistry

\section{INTRODUÇÃO}

A maioria das neoplasias primárias do trato gastrointestinal é maligna e pouco frequente, correspondendo aproximadamente a $2 \%$ das neoplasias caninas, e pode ser de origem epitelial, neuroendócrina, mesenquimal hematopoiética e mesenquimal não hematopoiética (Frost et al., 2003; Leandro e Sá, 2015). As neoplasias mesenquimais gastrointestinais não hematopoiéticas representam um grupo de neoplasias que têm como denominador comum a proliferação de células fusiformes ou epitelioides da parede do trato gastrointestinal (Head et al., 2003; Frost et al., 2003; Ceriel et al., 2007). Nesse grupo, estão incluídos os leiomiomas (LM), os leiomiossarcomas (LMS), o schwannoma (SCH), o hemangioma, o hemangiossarcoma, o lipoma, o lipossarcoma e os tumores estromais gastrointestinais (GIST) (Frost et al., 2003; Leandro e Sá, 2015). Devido

Recebido em 24 de setembro de 2015

Aceito em 1 de março de 2016

E-mail: liliansa@usp.br 
à elevada similaridade histomorfológica das neoplasias gastrointestinais mesenquimais, só é possível estabelecer o diagnóstico morfológico definitivo com a utilização da imunohistoquímica para caracterizar o fenótipo da célula neoplásica fusiforme (Frost et al., 2003). O GIST é a neoplasia mesenquimal originária de células mesenquimais primitivas com a capacidade de diferenciação pluripotencial, sendo positivo para o anticorpo C-KIT (CD117). São descritos quatro padrões morfológicos, embora possa ocorrer a mistura dos padrões em um mesmo tumor: estoriforme, mixoide, fascicular e epitelioide (Head et al., 2003). A diferenciação celular, o número de mitose e a extensão de áreas de necrose são variáveis que são utilizadas para definir se os GIST são benignos ou malignos (Head et al., 2003). Estudos ultraestruturais e imuno-histoquímicos revelaram que os GIST podem ser classificados segundo a diferenciação em músculo liso (miogênica), tecido neural (neurogênica), combinação de ambos (misto) ou indiferenciado (anaplásico), justificando seu difícil diagnóstico e comportamento imprevisível (Bettini et al., 2003). A literatura que reúna tanto características morfológicas como alterações clínicas e laboratoriais de cães é escassa, o que dificulta o diagnóstico e o prognóstico dessas neoplasias. Nesse sentido, o presente estudo buscou caracterizar, do ponto de vista clínico, laboratorial, anatomopatológico e imunohistoquímicos, três casos de GIST em cães.

\section{CASUÍSTICA}

Foi realizado estudo observacional retrospectivo, fundamentado em análise de prontuários de 62 cães com diagnóstico de neoplasia gastrointestinal, no período de 10 anos no Hospital Veterinário da Faculdade de Medicina Veterinária e Zootecnia da Universidade de São Paulo (Hovet-FMVZ-USP). Três casos foram diagnosticados com GIST, correspondendo a uma frequência de ocorrência de $4,9 \%$, cujo perfil epidemiológico e os dados clínicos do primeiro atendimento estão dispostos na Tab. 1, enquanto as informações laboratoriais referentes ao hemograma e à bioquímica sérica (função renal e função hepática) estão na Tab. 2. Os critérios diagnósticos de GIST se basearam em características histomorfológicas e imunohistoquímicas das neoplasias (Head et al., 2003; Ceriel et al., 2007). A descrição das características histopatológicas, tais como diferenciação tecidual e índice mitótico, está apresentada na Tab. 3. Os resultados imunohistoquímicos encontrados para confirmar o diagnóstico e determinar a diferenciação tumoral estão apresentados na Tab. 4.

Tabela 1. Distribuição dos dados epidemiológicos, clínicos e macroscópicos dos cães com tumor estromal gastrointestinal no primeiro atendimento

\begin{tabular}{|c|c|c|c|}
\hline Dados & Caso 1 & Caso 2 & Caso 3 \\
\hline Espécie & Canina & Canina & Canina \\
\hline Sexo & Macho & Macho & Macho \\
\hline Idade & 8 anos & 8 anos & 14 anos \\
\hline Peso & $12,7 \mathrm{~kg}$ & $27 \mathrm{~kg}$ & $16 \mathrm{~kg}$ \\
\hline Condição corpórea & Magro & Magro & Magro \\
\hline Queixa principal & Anorexia/melena & Anorexia/prostração & $\begin{array}{l}\text { Emagrecimento/ } \\
\text { prostração }\end{array}$ \\
\hline \multirow[t]{3}{*}{ Exame físico } & Mucosas hipocoradas & Mucosas hipocoradas & Mucosas hipocoradas \\
\hline & Desidratação & Dispneia & Sensibilidade abdominal \\
\hline & Formação mesogástrica & & Formação mesogástrica \\
\hline Localização & Jejuno & Ceco & Jejuno \\
\hline Tamanho do neoplasma & $11 \times 6.5 \times 5,2 \mathrm{~cm}$ & $16 \times 7.5 \times 6,2 \mathrm{~cm}$ & $10,5 \times 5,0 \times 3,5 \mathrm{~cm}$ \\
\hline Ruptura intestinal & Ausente & Ausente & Presente / peritonite \\
\hline Metástase & Ausente & Ausente & Ausente \\
\hline Tratamento & Enterectomia/anastomose & Enterectomia/anastomose & Enterectomia/anastomose \\
\hline Quimioterapia & Não realizado & Não realizado & Não realizado \\
\hline Sobrevida & 30 dias & 12 meses & $1 \mathrm{dia}$ \\
\hline
\end{tabular}


Tabela 2. Distribuição dos resultados laboratoriais dos animais portadores de tumor estromal gastrointestinal

\begin{tabular}{l|l|l|l|l}
\hline Exames laboratoriais & Caso 1 & Caso 2 & Caso 3 & Referências \\
\hline Eritrograma & & & & \\
Eritrócitos $\left(\mathrm{x} 10^{6} / \mu \mathrm{L}\right)$ & 4,2 & 4,9 & 1,6 & $5-8$ \\
Hematócrito $(\%)$ & 22 & 11 & 9 & $37-54$ \\
Hemoglobina $(\mathrm{g} / \mathrm{dL})$ & 7,3 & 11,2 & 3,1 & $12-18$ \\
VCM $\left(\mu^{3}\right)$ & 60 & 65 & 62 & $60-77$ \\
HCM $(\mathrm{pg})$ & 22 & 24 & 21 & $22-27$ \\
CHCM $(\%)$ & 28 & 30 & 24 & $31-36$ \\
\hline Leucograma & & & & \\
\hline Leucócitos $\left(/ \mu^{3}\right)$ & 49.600 & 22.000 & 42.000 & $6000-15000$ \\
Bastonetes $\left(/ \mu^{3}\right)$ & 0 & 0 & 0 & $0-300$ \\
Segmentados $\left(/ \mu^{3}\right)$ & 26.000 & 22.000 & 29.000 & $3000-11800$ \\
Linfócitos $\left(/ \mu^{3}\right)$ & 2000 & 1500 & 3000 & $1500-5000$ \\
Monócitos $\left(/ \mu^{3}\right)$ & 0 & 100 & 0 & $0-800$ \\
Eosinófilos $\left(/ \mu^{3}\right)$ & 0 & 0 & 0 & $0-750$ \\
Basófilos $\left(/ \mu^{3}\right)$ & 0 & 0 & 0 & $0-150$ \\
Plaquetas $\left(\mathrm{x} 10^{3} / \mu^{3}\right)$ & 455 & 258 & 865 & $200-500$ \\
Comentários & $\mathrm{a}, \mathrm{c}, \mathrm{e}$ & $\mathrm{a}, \mathrm{b}, \mathrm{c}, \mathrm{d}, \mathrm{e}$ & $\mathrm{a}, \mathrm{c}, \mathrm{e}$ & $\mathrm{s} / \mathrm{c}$ \\
\hline Perfil renal & & & & \\
\hline Ureia $(\mathrm{mg} / \mathrm{dL})$ & 29,9 & 24 & 25 & até 40 \\
Creatinina $(\mathrm{mg} / \mathrm{dL})$ & 0,63 & 1,3 & 1,0 & até 1,3 \\
\hline Perfil hepático & & & & \\
\hline Proteína total $(\mathrm{g} / \mathrm{dL})$ & 7,1 & 7,2 & 5,4 & $5,5-8,0$ \\
Albumina $(\mathrm{g} / \mathrm{dL})$ & 1,9 & 2,2 & 3,2 & $2,5-4,5$ \\
ALT $(\mathrm{U} / \mathrm{L}) *$ & 5,1 & 152 & 36,5 & até 50 \\
FA $(\mathrm{U} / \mathrm{L}) \#$ & 126 & 145 & 24,2 & até 130 \\
\hline Cong & & & & \\
\hline
\end{tabular}

Comentários: a) morfologia celular normal. b) discreta anisocitose, c) policromasia, d) anisocitose,

e) série branca morfologia normal. *Alanina aminotransferase U/L. "Fosfatase alcalina U/L.

$\mathrm{S} / \mathrm{C}$ sem comentário

Os fragmentos de intestino foram avaliados em microscópio de luz nos aumentos de 40x, 100x e 400x. Em todos os casos, a análise microscópica revelou proliferação de células fusiformes arranjadas em feixes longos densamente celulares (Fig. 1 e 2). As células neoplásicas apresentaram citoplasma de limites pouco definidos, eosinofílico a basofílico, núcleo ovalado a alongado, com cromatina granular e nucléolo evidente único e grande ou pequeno e duplo. A Tab. 3 apresenta as demais características histopatológicas de cada caso.

Os fragmentos neoplásicos foram submetidos ao exame imuno-histoquímico para caracterização imunofenotípica da célula neoplásica. $\mathrm{O}$ método empregado foi estreptavidina-biotina-peroxidase. Os principais marcadores e seus respectivos códigos e diluições utilizados foram vimentina (Cód. M0725, 1:100); actina músculo liso (Cód. M0851,1:2500); desmina (Cód. M0760, 1:250);
S100 (Cód. Z0311, 1:10.000); CD117 (Cód. A4502, 1:500); CD34 (Cód. M7165, 1:6000); citoqueratina AE1 $\backslash$ AE3 (Cód. M3515, 1:100). Em todas as amostras, foram realizados controles negativos e positivos. Tumor estromal gastrointestinal humano foi o controle positivo para C-KIT (CD117). O segmento de intestino canino normal foi usado como controle positivo para os marcadores mesenquimais. Para a actina do músculo liso e a desmina, o controle positivo utilizado foi o muscular da mucosa e o tecido muscular liso na parede vascular. As células endoteliais vasculares foram usadas como controle positivo para a vimentina, enquanto o plexo mioentérico foi o controle para S100. Para citoqueratina, o epitélio intestinal foi o controle positivo. A Tab. 4 apresenta os resultados imuno-histoquímicos e o diagnóstico final: GIST com diferenciação neurogênica (caso 1), miogênica (caso 2) e anaplásico (caso 3). 

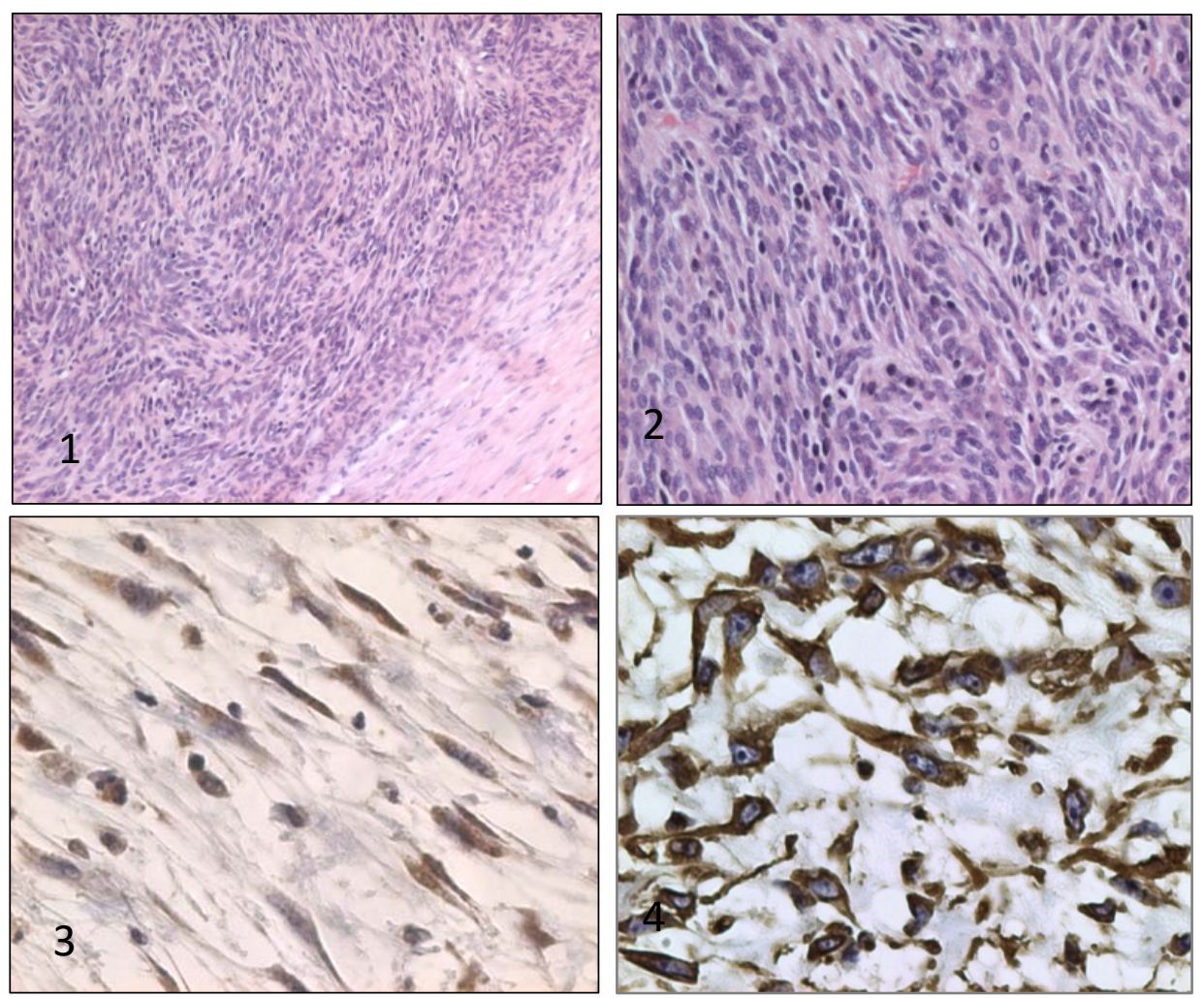

Figura 1. Caso 1 - proliferação de células fusiformes arranjadas em feixes longos densamente celulares, HE, 40x. Figura 2. Caso 3 - células neoplásicas com citoplasma de limites pouco definidos, núcleo ovalado a alongado com nucléolo evidente, pleomorfismo nuclear elevado e anisocariose e aumento da relação núcleo:citoplasma. HE, 100x. Figura 3. Caso 1- imunomarcação citoplasmática para CD117, HE, 400x. Figura 4. Caso 1 - imunomarcação citoplasmática para vimentina, HE, 400x.

Tabela 3. Principais características histopatológicas observadas nos casos estudados de tumor estromal gastrointestinal em cães

\begin{tabular}{llll}
\hline Características histopatológicas & Caso 1 & Caso 2 & Caso 3 \\
\hline Padrão & Fascicular & Fascicular & Fascicular \\
Diferenciação tecidual & Pouco diferenciado & Pouco diferenciado & Moderada atipia \\
Índice mitótico & 20 por campo de 400x & $0-9$ por campo de 400x & 0-9 por campo de 400x \\
Área de necrose & Maior do que 50\% & Maior do que 50\% & Menor do que 50\% \\
Ulceração & Presente & Presente & Presente \\
Hemorragia & Ausente & Presente & Ausente \\
Camadas comprometidas & Todas as camadas & Camada muscular & Todas as camadas \\
Invasão vascular & Ausente & Ausente & Ausente \\
Relação núcleo:citoplasma & & Aumentado & \\
Pleomorfismo nuclear & Ausente & Presente & Ausente \\
Megacariose & Ausente & Presente & Ausente \\
Anisiocariose & Ausente & Presente & Ausente \\
\hline
\end{tabular}

${ }^{\#}$ Maior ou menor que $50 \%$ da superfície da neoplasia. 
Tabela 4. Resultado imuno-histoquímico e diagnóstico morfológico final segundo cada caso

\begin{tabular}{lllllllll}
\hline Caso & Diagnóstico & Vimentina & CD117 & S100* & SMA $^{\#}$ & Desmina & CD34 & Citoqueratina \\
\hline 1 & GIST neurogênica & + & + & + & - & - & - & - \\
2 & GIST miogênica & + & + & - & + & - & - & - \\
3 & GIST anaplásico & + & + & - & - & - & - & - \\
\hline
\end{tabular}

\section{DISCUSSÃO}

Os estudos que englobam as associações clínicolaboratorial, epidemiológica e anatomopatológica de pacientes com GIST, aliadas aos aspectos imuno-histoquímicos, são poucos, embora a frequência de ocorrência de neoplasias gastrointestinais em cães aumente em animais de meia-idade e idosos (LaRock e Ginn, 1997; Frost et al., 2003; Ceriel et al., 2007; Russel et al., 2007).

Não há estudos veterinários que abordem a frequência de ocorrência do GIST em cães, mas, no contexto desta análise, a casuística foi $4,9 \%$ em 10 anos de levantamento. Em seres humanos, os GIST são as neoplasias mesenquimais mais comuns do trato gastrointestinal, com uma incidência de um a dois casos por 100.000 indivíduos em todo o mundo (Tran et al., 2005).

O perfil epidemiológico dos cães acometidos se caracteriza por estes serem machos, adultos, com idade média de nove anos, de médio porte e com peso médio de $18,5 \mathrm{~kg}$, similar às características apontadas em cães com neoplasias gastrointestinais, como GIST, na literatura (Ceriel et al., 2007; Leandro e Sá, 2015). Os sinais clínicos observados em todos os casos se caracterizam por alterações crônicas gastrointestinais, como melena, e extragastrointestinais inespecíficas, como prostração, anorexia e perda de peso, como já descritos (Russel et al., 2007). Geralmente as alterações clínicas são incipientes e progressivas, o que retarda o diagnóstico clínico-cirúrgico.

Anemia não regenerativa e leucocitose foram as principais alterações laboratoriais encontradas em todos os casos. A literatura aponta que a anemia é a alteração laboratorial mais comum, acometendo $33 \%$ dos animais com neoplasias gastrointestinais. Tal alteração é secundária à perda de sangue intestinal crônica, pela evolução e duração da doença (Leandro e Sá, 2015). A leucocitose e a presença de neutrófilos tóxicos, como foi observado, podem estar associadas ao quadro de bacteremia secundária à ulceração da mucosa intestinal e à necrose tumoral (Russel et al., 2007; Leandro e Sá, 2015). As alterações bioquímicas - função hepática, renal, proteína total e albumina - são variáveis inespecíficas nos quadros de neoplasia gastrointestinal e não ajudam no diagnóstico de processo neoplásico gastrointestinal, como nos casos de GIST.

A avaliação imuno-histoquímica é extremamente importante e mandatória para predizer a origem tumoral dos sarcomas fusiformes gastrointestinais, bem como diferenciar o GIST (vimentina,$+ \quad \mathrm{CD} 117+$ ) das verdadeiras neoplasias de músculo liso representadas pelos leiomiossarcomas e leiomiomas (vimentina +, SMA +, desmina +/-, CD117-), e sarcomas indiferenciados (vimentina +, S100-, CD117-, SMA-, desmina -, CD117-) (LaRock e Ginn, 1997; Frost et al., 2003; Ceriel et al., 2007; Russel et al., 2007). Em todos os casos analisados, o diagnóstico de GIST se baseou na imunomarcação positiva para vimentina e CD117. Os GIST são neoplasias $100 \%$ positivos para vimentina e negativos para citoqueratina e proteína glial fibrilar ácida (Reis-Filho et al., 2003; Bettini et al., 2003; Ceriel et al., 2007). O marcador imuno-histoquímico CD117 apresenta marcação citoplasmática e está presente em até 54 - 58\% dos GIST malignos em cães, sendo pouco frequente nas formações benignas $\mathrm{e}$ ausente nas neoplasias típicas de origem muscular e nervosa (Bettini et al., 2003; ReisFilho et al., 2003; Russel et al., 2007). Em casos de GIST CD117 negativos, uma opção para se obter o diagnóstico definitivo seria a utilização do DOG 1; este marcador tem boa especificidade e sensibilidade para o diagnóstico de GIST canino, embora, nos casos estudados, não tenha sido utilizado (Dailey et al., 2015). A inclusão do marcador DOG1 em painéis imunohistoquímicos junto com marcador CD117 irá melhorar o diagnóstico e a precisão do diagnóstico do GIST canino, especialmente naqueles em que CD117 é negativo. Como o GIST pode apresentar diferenciação miogênica, neurogênica, mista ou ser anaplásico, a análise 
dos demais marcadores, como desmina, actina de músculo liso, S100 e CD34, foi realizada para determinar a possível diferenciação das células neoplásicas.

Assim, o caso 1 apresentou imunomarcação também para $\mathrm{S} 100$. A proteína $\mathrm{S} 100$ é um marcador típico das lesões originárias do plexo nervoso mioentérico, que está presente, em maior parte, nas neoplasias de origem nervosa e ausente nas neoformações de origem muscular. São positivos para S100 68 a 73\% dos GIST, caracterizando diferenciação neurogênica (Bettini et al., 2003; Frost et al., 2003).

No caso 2, houve marcação positiva para o anticorpo SMA, confirmando diferenciação miogênica. A imunoexpressão positiva de SMA e a de desmina são observadas em células normais de músculo liso e em células neoplásicas de músculo liso, benignas ou malignas. SMA é positiva em aproximadamente $33-40 \%$ dos GIST caninos, enquanto a desmina é positiva entre 80 82\% (Reis-Filho et al., 2003; Bettini et al., 2003; Ceriel et al., 2007; Russel et al., 2007).

A classificação anaplásica descrita no caso 3 foi baseada na imunomarcação positiva para CD117 e negativa para os anticorpos SMA, desmina e S100 (Ceriel et al., 2007), não sendo, dessa forma, possível determinar a diferenciação das células neoplásicas. Em nenhum dos casos, houve marcação para CD34. Mas o CD34 pode ser expresso em 14,8\% dos GIST caninos (Gillespie et al., 2011). Em seres humanos, 60$70 \%$ de todos os GIST localizados no esôfago e no reto são positivos para $\mathrm{CD} 34$, não havendo diferença significativa da expressão do CD34 entre os GIST benignos e malignos (Gillespie et al., 2011). GIST estão localizados principalmente no intestino grosso, em $48 \%$ dos casos, especialmente no ceco, e $29 \%$ dos casos no intestino delgado, com maior frequência no jejuno (Frost et al., 2003; Ceriel et al., 2007); nos cães analisados, dois apresentaram GIST no intestino delgado (casos 1 e 3 ) e um no ceco (caso 2). Os GIST geralmente são grandes, com medidas que variam de $2,5 \mathrm{~cm}$ a $30 \mathrm{~cm}$ de diâmetro, com superfície irregular, comprometendo principalmente a camada muscular e a mucosa do órgão. Os achados clínicos, laboratoriais e anatomopatológicos do presente estudo estão em concordância com a literatura em relação à localização e ao tamanho da neoformação. Aqui, os GIST mediram, em média, $19 \mathrm{~cm}$ no maior eixo e comprometiam todas as camadas da parede intestinal em, pelo menos, dois cães (casos 1 e 3). O índice mitótico é bastante variável nos GIST caninos, em que $65 \%$ dos animais apresentam de uma a 19 mitoses em 10 campos de 400x e $43 \%$ apresentam áreas de necrose (Frost et al.,2003; Ceriel et al., 2007; Russel et al., 2007). Os casos abordados exibem índice mitótico variável e extensas áreas de necrose, como nos casos 1 e 2. As alterações histomorfológicas descritas enfatizam que os GIST analisados foram casos malignos, o que pode estar associado à baixa sobrevida após o diagnóstico e a intervenção cirúrgica de um dia (caso 3, com peritonite) a 12 meses. Em nenhum caso foi observada metástase a distância. Essas variáveis, como tamanho, juntamente com o elevado índice mitótico, necrose e localização no ceco, constituem os mais fidedignos parâmetros para se estimar agressividade, bem como associação ao maior risco de metástase, perfuração intestinal e, consequentemente, desenvolvimento de peritonite e septicemia (Reis-Filho et al., 2003; Bettini et al., 2003; Ceriel et al., 2007). A sobrevida dos animais avaliados é similar aos dados da literatura, em que a sobrevida média de animais com GIST submetidos a procedimento cirúrgico, sem tratamento quimioterápico adjuvante, reporta 11,6 meses (Cohen et al, 2003).

O GIST deve ser sempre um dos diagnósticos diferenciais a serem considerados entre as neoplasias mesenquimais do trato gastrointestinal de cães, e para o diagnóstico final, a caracterização imuno-histoquímica é de suma importância.

\section{REFERÊNCIAS}

BETTINI, G.; MORINI, M.; MARCATO, P.S. Gastrointestinal spindle cell tumours of the dog: histological and immunohistochemical study. $J$. Comp. Pathol., v.129, p.283-293, 2003.

CERIEL, P.H.J.M.; TER HAAR, G.; VAN DER GAGA, I.; KIRPENSTEIJN, J. Reclassification of small intestinal and cecal smooth muscle tumors in 72 dogs: clinical, histologic, and immunohistochemical evaluation. Vet. Surg., v.36, p.302-313, 2007. 
COHEN, M.; POST, G.S.; WRIGHT, J.C. Gastrointestinal leiomyosarcoma in 14 dogs. $J$. Vet. Int. Med., v.17, p.107-110, 2003.

DAILEY, D.D.; EHRHART, E.J.; DUVAL, D.L. et al. Dog1 is a sensitive and specific immunohistochemical marker for diagnosis of canine gastrointestinal stromal tumors. J. Vet. Diag. Invest., v.27, p.268-277, 2015.

FROST, D.; LASOTA, J., MIETTINEN, M. Gastrointestinal stromal tumors and leiomyomas in the dog: a histopathologic, immunohistochemical, and molecular genetic study of 50 cases. Vet. Pathol., v.40, p.42-54, 2003.

GILLESPIE, V.; BAER, K.; FARRELLY, J. et al. Canine gastrointestinal stromal tumors: immunohistochemical expression of CD34 and examination of prognostic indicators including proliferation markers Ki67 and AgNOR. Vet. Pathol., v.48, p.283-291, 2011.

HEAD, K.W.; CULLEN, J.M.; DUBIELZIG, R.R. Histological classification of tumors of the intestines in domestical animals. In: HEAD, K.W.; CULLEN, J.M.; DUBIELZIG, R.R. (Eds.). Histological classification of tumors of the alimentary system of domestic animals. Washington: Armed Forces Institute of Pathology, 2003. v.10, p.87-100.
LA ROCK, P.G.; GINN, P.E. Immunuhistochemical staining characteristics of canine stromal tumors. Vet. Pathol., v.34, p.303331, 1997.

LEANDRO, R.M.; SÁ, L.R.M. Neoplasias gastrointestinais. In: JERICÓ, M.M.; ANDRADE NETO, J.P.; KOGIKA, M.M. (Eds.). Tratado de medicina interna de cães $e$ gatos. Rio de Janeiro: Rocca, 2015. p.995-1003.

REIS-FILHO, J.S.; GONCALVES, C.; GARTNER, F. Rectal leiomyosarcoma in a dog and review of gastrointestinal stromal tumours. Vet. Rec., v.153, p.215-217, 2003.

RUSSEL, K.R.; MEHLER, S.J.; SKORUPSKI, K.A. et al. Clinical and immunohistochemical differentiation of gastrointestinal stromal tumors from leiomyosarcomas in dogs: 42 cases (19902003). J. Am. Vet. Med. Assoc., v.230, p.13291333, 2007.

TRAN, T.; DAVILA, J.A.; EL-SERAG, H.B. The epidemiology of malignant gastrointestinal stromal tumors: an analysis of 1,458 cases from 1992 to 2000. Am. J. Gastroenterol., v.100, p.162-168, 2005. 